Received: 2017.03.14

Accepted: 2017.05.16

Published: 2017.09.08

Authors' Contribution:

Study Design A

Data Collection B

Statistical Analysis C

Data Interpretation D

Manuscript Preparation E

Literature Search $F$

Funds Collection G

Corresponding Author:

Conflict of interest:

ABEF Filippo Mearelli

DE Chiara Casarsa

E Andrea Breglia

E Gianni Biolo

None declared

\title{
Septic Shock with Multi Organ Failure Due to Fluoroquinolones Resistant Campylobacter Jejuni
}

Department of Medical Surgical and Health Sciences, Internal Medicine Division Cattinara Hospital, University of Trieste, Trieste, Italy

Filippo Mearelli, e-mail: filippome@libero.it

Patient: Male, 65

Final Diagnosis: $\quad$ Septic shock with MOF due to fluoroquinolones resistant Campylobacter jejuni

Symptoms: $\quad$ Fever $\left(37.4^{\circ} \mathrm{C}\right) \bullet$ acute delirium

Medication: -

Clinical Procedure: -

Specialty: Infectious Diseases

Objective: Rare disease

Background: Campylobacter jejuni infections are typically self-limited, and severe extra-intestinal complications are uncommon.

Case Report: We report a case of a man with septic shock due to fluoroquinolones resistant Campylobacter jejuni.

Conclusions: $\quad$ This manuscript emphasizes the potential lethality of fluorquinolones resistant Campylobacter jejuni bacteremia.

MeSH Keywords: Campylobacter Jejuni • Fluoroquinolones • Shock, Septic

Full-text PDF: https://www.amjcaserep.com/abstract/index/idArt/904337

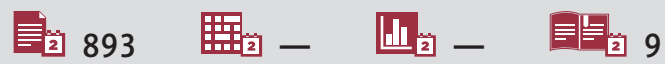




\section{Background}

Campylobacter spp infections are typically self-limited and severe complications are quite rare. Bacteremia caused by Campylobacter spp has been detected in less than $1 \%$ of patients with gastroenteritis due to these species. Attributable mortality of Campylobacter spp bacteremia has been estimate to be $4-16 \%$. The impact of appropriateness of antimicrobial therapy on outcomes is controversial. Moreover, in the last 20 years, the incidence of fluoroquinolone resistance has been increasingly reported: in 2007, in the USA, it peaked at nearly $26 \%$ of human isolates.

\section{Case Report}

In May 2014, a 65-year-old male presented to us with low grade fever $\left(37.4^{\circ} \mathrm{C}\right)$ and acute delirium. Past medical history was significant for HCV cirrhosis (Child-Pugh class C) and betalactam allergy. Clinical examination found hypotension (60/40 mm Hg), tachycardia (115/minutes), tachypnea (35/minutes), ascites with abdominal tenderness, and signs of portal-systemic encephalopathy.

Blood tests showed leukocytosis $(19,000 / \mu \mathrm{L})$, acute decompensation of liver function (AST $141 \mathrm{U} / \mathrm{L}$, ALT $49 \mathrm{U} / \mathrm{L}$, total bilirubin $12 \mathrm{mg} / \mathrm{dL}$ ), disseminated intravascular coagulation (platelets $58,000 / \mathrm{mm}^{3}$, PT and aPTT incoagulable, fibrinogen $30 \mathrm{mg} / \mathrm{dL}$, D-dimer 36 ), and acute renal failure (increase in serum creatinine of $0.3 \mathrm{mg} / \mathrm{dL}$ from baseline within 48 hours from admission). His ammonium level was $86 \mu \mathrm{g} / \mathrm{dL}$ and HIV serology was negative. Thirty minutes after admission, it was decided to start the patient on empirical antibiotic treatment with intravenous ciprofloxacin $200 \mathrm{mg}$ twice daily on the suspicion of spontaneous bacterial peritonitis. Lactulose enemas and parenteral branched-chain amino acids were also administered.

A Swan-Ganz catheter was not inserted due to coagulation disorders despite fresh frozen plasma administration. Ultrasoundguided resuscitation with crystalloids and norepinephrine was useful in achieving mean blood pressure over $65 \mathrm{~mm} \mathrm{Hg}$, adequate urine output and optimal lactate clearance. At 48 hours after admission, the patient was able to maintain stable vital signs; a total body computed topography (CT) was performed to rule out ongoing hemorrhages, pneumonia, choledocholithiasis, and abscesses. Twenty-four hours later, catecholamines were gradually interrupted and two blood culture specimens yielded Campylobacter jejuni resistant to fluoroquinolones and sensitive to macrolides.

Blood cultures were tested using BACTEC blood culture system according to the manufacturer's instructions. Spiral shaped or curved Gram negative rods identified from blood cultures that formed distinct colony morphology were further processed to campylobacter species using API Campy system. The isolate was tested for susceptibility to aminoglycosides, macrolides, and fluoroquinolones by means of the standard agar disk diffusion method.

\section{Urine and stools remained negative}

Because of antimicrobial susceptibility results and the patient's improvement and ability to take oral medication, empirical therapy was shifted to azithromycin 500 mg orally daily. For the same reason, lactulose enemas were suspended and the patient was commenced on lactulose oral solution.

One week after admission, the patient's acute decompensation of liver function, disseminated intravascular coagulation, and acute renal failure resolved. The patient was discharged one week later.

\section{Discussion}

Campylobacter jejuni is a microaerophilic, gram negative rod. Most frequently infections caused by Campylobacter jejuni manifest as a self-limited diarrheal illness with an associated abdominal pain.

In Europe, bacteremia due to Campylobacter spp was detected in less than $1 \%$ of the patients with gastroenteritis [1]. The low rate of detection could partly be explained by under diagnosis due to bactericidal properties of human serum against the species [2]. Another possible explanation is that blood cultures are not routinely performed for acute gastroenteritis, even when patients are febrile [2].

Campylobacter jejuni is the most frequently isolated species causing sepsis [1]. Septicemia occurs mostly among immunocompromised patients (mainly AIDS) or those with other comorbidities (malignancies and liver disease) [3]. In Italy, evidence is limited to some case reports $[2,4]$.

The absence of a portal of origin is documented in less than $30 \%$ of the patients [1].

In the Pigrau et al. case series, only 1 out of 47 bacteremia cases were due Campylobacter jejuni developed septic shock [5]. Even if case fatality rate due to Campylobacter jejuni bacteremia is low, around $10 \%$ (but may be higher in HIV infected patients) [1], blood stream infections associated with high Pittsburgh Bacteremia Score can cause death [6,7]. In a recent retrospective study the mortality attributable to septic shock caused by Campylobacter jejuni was high (4 out of 4 cases died) [1]. In our patient, the severity of infection could have 
been enhanced by his immunosuppression induced by cirrhosis. Fluoroquinolones resistance has been documented; and it is increasingly common in some countries such as Spain [1] and Taiwan [8] where these drugs should not be considered for empirical therapy. In addition, emergence of resistance could represent an important issue for returning traveler's diarrhea.

Nevertheless, in patients with Campylobacter spp bacteremia, the impact of an appropriate treatment on prognosis continues to be controversial and there is a lack of evidence, especially for severe infections [1]. Pacanowsky et al. reported that failure to administer appropriate antibiotics in bacteremia caused by Campylobacter spp was associated with fatal outcome [9].

However, in two recent Spanish [1] and Finnish [3] retrospective studies, inappropriate antimicrobial therapy did not alter

\section{References:}

1. Fernández-Cruz A, Muñoz P, Mohedano R et al: Campylobacter bacteremia: Clinical characteristics, incidence, and outcome over 23 years. Medicine (Baltimore), 2010; 89(5): 319-30

2. Gallo MT, Di Domenico EG, Toma L et al: Campylobacter jejuni fatal sepsis in a patient with non-Hodgkin's lymphoma: Case report and literature review of a difficult diagnosis. Int J Mol Sci, 2016; 17(4): 544

3. Feodoroff B, Lauhio A, Ellström P, Rautelin H: A Nationwide study of Campylobacter jejuni and Campylobacter coli bacteremia in Finland over a 10-year period, 1998-2007, with special reference to clinical characteristics and antimicrobial susceptibility. Clin Infect Dis, 2011; 53(8): e99-e106

4. Manfredi R, Maietti A, Ferri M, Chiodo F: Fatal Campylobacter jejuni bacteremia in patients with AIDS. J Med Microbiol, 1999; 48: 601-3 outcomes. More studies are needed to determine the impact of appropriateness of therapy on mortality.

\section{Conclusions}

The presented case of septic shock caused by fluoroquinoloneresistant Campylobacter jejuni on one hand was treated with an inappropriate antibacterial therapy and on the other hand was treated with a timely point of care multi-organ ultrasoundguided resuscitation. We feel that the latter aspects could have contributed significantly to positive outcome.

\section{Conflicts of interest}

None.

5. Pigrau C, Bartolome R, Almirante B et al: Bacteremia due to Campylobacter species: Clinical findings and antimicrobial susceptibility patterns. Clin Infect Dis, 1997; 25(6): 1414-20

6. Meyrieux V, Monneret G, Lepape A et al: Fatal septic shock with multiple organ failure due to Campylobacter jejuni. Clin Infect Dis, 1996; 22(1): 183-84

7. Eltawansy SA, Merchant C, Atluri P, Dwivedi S: Multi-organ failure secondary to a Clostridium perfringens gaseous liver abscess following a self-limited episode of acute gastroenteritis. Am J Case Rep, 2015; 16: 182-86

8. Liao $\mathrm{CH}$, Chuang CY, Huang YT et al: Bacteremia caused by antimicrobial resistant Campylobacter species at a medical center in Taiwan, 1998-2008. J Infect, 2012; 65(5): 392-99

9. Pacanowski J, Lalande V, Lacombe K et al: Campylobacter bacteremia: Clinica features and factors associated with fatal outcome. Clin Infect Dis, 2008; 47: 790-96 